\title{
INTEGRATION WITH RESPECT TO LOCAL TIME AND ITÔ'S FORMULA FOR SMOOTH NONDEGENERATE MARTINGALES
}

\author{
Xavier Bardina and Carles Rovira
}

Abstract

We show an Itô's formula for nondegenerate Brownian martingales $X_{t}=\int_{0}^{t} u_{s} d W_{s}$ and functions $F(x, t)$ with locally integrable derivatives in $t$ and $x$. We prove that one can express the additional term in Itô's s formula as an integral over space and time with respect to local time.

\section{Introduction}

We consider a continuous nondegenerate martingale $X=\left\{X_{t}, t \in\right.$ $[0,1]\}$ of the form $X_{t}=\int_{0}^{t} u_{s} d W_{s}$ where $W=\left\{W_{t}, t \in[0,1]\right\}$ is a standard Brownian motion and $u$ is an adapted stochastic process. Let $F: \mathbb{R} \times[0,1] \rightarrow \mathbb{R}$ be an absolutely continuous function with partial derivatives satisfying some local integrability properties. The main aim of this paper is to obtain an Itô's formula for $F\left(X_{t}, t\right)$ where the term corresponding usually to the second order derivative is expressed as an integral over space and time with respect to local time.

We will prove this results when $u$ satisfies (locally) the assumptions (H1) For all $t \in[0,1], u_{t}$ belongs to the space $\mathbb{D}^{3,2}$ and for all $p \geq 2$

$$
\begin{aligned}
E\left|u_{t}\right|^{p}+E\left|D_{s} u_{t}\right|^{p}+E( & \left.\int_{r \vee s}^{1}\left|D_{r} D_{s} u_{\theta}\right|^{2} d \theta\right)^{p / 2} \\
& +E\left(\int_{r \vee s \vee v}^{1}\left|D_{v} D_{r} D_{s} u_{\theta}\right|^{2} d \theta\right)^{p / 2} \leq K_{p},
\end{aligned}
$$

(H2) $\left|u_{t}\right| \geq \rho>0$ for some constant $\rho$ and for all $t \in[0,1]$.

Moret and Nualart [14] consider an Itô's formula for this class of nondegenerate martingales. Their main result reads as follows:

2000 Mathematics Subject Classification. 60H05, 60G44.

Key words. Martingales, integration wrt local time, Itô's formula, local time. 
Theorem 0.1 (Moret and Nualart, [14]). Let $u$ be a process satisfying (H1) and (H2). Set $X_{t}=\int_{0}^{t} u_{s} d W_{s}$. Then for any function $f \in L_{\text {loc }}^{2}(\mathbb{R})$ the quadratic covariation $[f(X), X]$ exists and the following Itô's formula holds

$$
F\left(X_{t}\right)=F(0)+\int_{0}^{t} f\left(X_{s}\right) d X_{s}+\frac{1}{2}[f(X), X]_{t},
$$

for all $t \in[0,1]$, where $F(x)=F(0)+\int_{0}^{x} f(y) d y$.

Moret [13] gave an extension of this last result for functions $F$ depending also on $t$. She considers a new hypothesis on functions $f$ :

(C) $f(\cdot, t) \in L_{\text {loc }}^{2}(\mathbb{R})$ and for all compact set $K \subset \mathbb{R}, f(x, t)$ is continuous in $t$ as a function of $[0,1]$ to $L^{2}(K)$.

Then, her result is the following:

Theorem 0.2 (Moret, [13]). Let u be a process satisfying (H1) and (H2). Set $X=\int_{0}^{t} u_{s} d W_{s}$. Let $F(x, t)$ be an absolutely continuous function in $x$ such that the partial derivative $f(\cdot, t)$ satisfies $(C)$. Then, the quadratic covariation $[f(X, \cdot), X]$ exists and the following Itô's formula holds

$$
F\left(X_{t}, t\right)=F(0,0)+\int_{0}^{t} f\left(X_{s}, s\right) d X_{s}+\frac{1}{2}[f(X, \cdot), X]+\int_{0}^{t} F\left(X_{s}, d s\right),
$$

where

$$
\int_{0}^{t} F\left(X_{s}, d s\right) \equiv \lim _{n \rightarrow+\infty} \sum_{t_{i} \in D_{n}, t_{i} \leq t}\left(F\left(X_{t_{i+1}}, t_{i+1}\right)-F\left(X_{t_{i+1}}, t_{i}\right)\right),
$$

exists uniformly in probability for $\left(D_{n}\right)_{n}$ a sequence of smooth partitions of $[0,1]$.

In these two results, following the ideas of Föllmer, Protter and Shiryayev [11] for the Brownian motion, the additional term is written as a quadratic covariation. Bardina and Jolis $[\mathbf{2}],[\mathbf{3}]$ extended the results of Föllmer et al. [11] to the case of the elliptic and hypoelliptic diffusions.

Nevertheless, it is important to point out the differences between [14] and $[\mathbf{1 1}]$. One of the keys of their proofs is to obtain some a priori estimates on the Riemann sums. In [11] these estimates are obtained using the semimartingale expression of the time-reversed Brownian motion and well-known bounds for the density of the Brownian motion. Moret and Nualart [14] used another approach, using Malliavin calculus in order to obtain sharp estimates for the density of the process $X_{t}$ and avoiding the time-reversed arguments. 
We want to express the quadratic variation term as an integral with respect to the local time. We will refer the reader to the book of Rogers and Williams $[\mathbf{1 7}]$ for the theory of local times for martingales.

There are several papers where the integrals with respect to local time are used in Itô's formula. In 1981, Bouleau and Yor [5] obtained the following extension of the Itô's formula:

Theorem 0.3 (Bouleau and Yor, [5]). Let $X=\left(X_{t}\right)_{t \geq 0}$ be a continuous semimartingale and let $F: \mathbb{R} \rightarrow \mathbb{R}$ be an absolutely continuous function with derivative $f$. Assume that $f$ is a mesurable locally bounded function. Then:

$$
F\left(X_{t}\right)=F\left(X_{0}\right)+\int_{0}^{t} f\left(X_{s}\right) d X_{s}-\frac{1}{2} \int_{\mathbb{R}} f(x) d_{x} L_{t}^{x}
$$

where $d_{x} L_{t}^{x}$ is an integral with respect to $x \rightarrow L_{t}^{x}$.

Eisenbaum [8], [9] defined an integral in time and space with respect to the local time of the Brownian motion. Using this integral, the quadratic covariation in the formula given in [11] can be expressed as an integral with respect to the local time. She obtained the following result:

Theorem 0.4 (Eisenbaum, $[\mathbf{8}]$ and $[\mathbf{9}]$ ). Let $W=\left(W_{t}\right)_{0 \leq t \leq 1}$ be a standard Brownian motion and $F$ a function defined on $\mathbb{R} \times[0,1]$ such that there exist first order Radon-Nikodym derivatives $\frac{\partial F}{\partial t}$ and $\frac{\partial F}{\partial x}$ such that for every $A \in \mathbb{R}_{+}$,

$$
\int_{0}^{1} \int_{-A}^{A}\left|\frac{\partial F}{\partial t}(x, s)\right| \frac{1}{\sqrt{s}} d x d s<+\infty
$$

and

$$
\int_{0}^{1} \int_{-A}^{A}\left(\frac{\partial F}{\partial x}(x, s)\right)^{2} \frac{1}{\sqrt{s}} d x d s<+\infty .
$$

Then,

$$
\begin{aligned}
F\left(W_{t}, t\right)=F\left(W_{0}, 0\right)+\int_{0}^{t} \frac{\partial F}{\partial x}\left(W_{s}, s\right) d W_{s} & +\int_{0}^{t} \frac{\partial F}{\partial t}\left(W_{s}, s\right) d s \\
& -\frac{1}{2} \int_{0}^{t} \int_{\mathbb{R}} \frac{\partial F}{\partial x}(x, s) d L_{s}^{x} .
\end{aligned}
$$

This result has been extended by Bardina and Rovira [4] for elliptic diffusion processes.

In our paper we will follow the ideas of Eisenbaum [8], [9], assuming on the function $F$ the hypothesis considered in Theorem 0.4. In the papers of Eisenbaum [8], [9], as well as in [11] or in the extension of Bardina 
and Rovira [4], one of the main ingredients is the study of the time reversed process and the relationship between the quadratic covariation and the forward and backward stochastic integrals. We show that we can adapt the methods of Eisenbaum without using the time reversed process and the backward integral. We will follow the methods of Moret and Nualart [14] and we will use Malliavin calculus to obtain the necessary estimates for the Riemann sums.

The existence of the quadratic covariation is not one of our main objectives. Nevertheless, it will be an important tool in our computations. We recall its definition.

Definition 1. Given two stochastic processes $Y=\left\{Y_{t}, t \in[0,1]\right\}$ and $Z=\left\{Z_{t}, t \in[0,1]\right\}$ we define their quadratic covariation as the stochastic process $[Y, Z]$ given by the following limit in probability, if it exists,

$$
[Y, Z]_{t}=\lim _{n} \sum_{t_{i} \in D_{n}, t_{i}<t}\left(Y_{t_{i+1}}-Y_{t_{i}}\right)\left(Z_{t_{i+1}}-Z_{t_{i}}\right)
$$

where $D_{n}$ is a sequence of partitions of $[0,1]$ whose mesh tends to 0 as $n$ goes to $\infty$.

We will assume that the partitions $D_{n}$ satisfy

(M) $\lim _{n} \sup _{t_{i} \in D_{n}}\left(t_{i+1}-t_{i}\right)=0, \quad M:=\sup _{n} \sup _{t_{i} \in D_{n}} \frac{t_{i+1}}{t_{i}}<\infty$.

We impose this condition in order to avoid certain possibly exploding Riemann sums.

Other extensions for Itô's formula has been obtained recently. Among others, there is the paper of Dupoiron et al. [7] for uniformly elliptic diffusions and Dirichet processes, the work of Ghomrasni and Peskir [12] for continuous semimartingales, the paper of Flandoli, Russo and Wolf [10] for a Lyons-Zheng process or the work of Di Nunno, Meyer-Brandis, Øksendal and Proske [6] for Lévy processes.

The paper is organized as follows. In Section 1 we give some basic definitions and results on Malliavin calculus, recalling some results obtained in [14]. In Section 2 we define the space where we are able to construct an integral in the plane with respect to the local time of a nondegenerate Brownian martingale. Finally, Section 3 is devoted to present our main result the extension of Itô's formula.

Along the paper we will denote all the constants by $C, C_{p}, K$ or $K_{p}$, unless they may change from line to line. 


\section{Preliminaries}

Let $(\Omega, \mathcal{F}, P)$ be the canonical probability space of a standard Brownian motion $W=\left\{W_{t}, 0 \leq t \leq 1\right\}$, that is, $\Omega$ is the space of all continuous functions $\omega:[0,1] \rightarrow \mathbb{R}$ vanishing at $0, P$ is the standard Wiener measure on $\Omega$ and $\mathcal{F}$ is the completion of the Borel $\sigma$-field of $\Omega$ with respect to $P$.

Let $\mathcal{S}$ be the set of smooth random variables of the form

$$
F=f\left(W_{t_{1}}, \ldots, W_{t_{n}}\right),
$$

$f \in \mathcal{C}_{b}^{\infty}\left(\mathbb{R}^{n}\right)$ and $t_{1}, \ldots, t_{n} \in[0,1]$. The Malliavin derivative of a smooth random variable $F$ of the form (1) is the stochastic process $\left\{D_{t} F, t \in\right.$ $[0,1]\}$ given by

$$
D_{t} F=\sum_{i=1}^{n} \frac{\partial f}{\partial x_{i}}\left(W_{t_{1}}, \ldots, W_{t_{n}}\right) I_{\left[0, t_{i}\right]}(t), \quad t \in[0,1] .
$$

The Malliavin derivative of order $N \geq 2$ is defined by iteration, as follows. For $F \in \mathcal{S}, t_{1}, \ldots, t_{N} \in[0,1]$,

$$
D_{t_{1}, \ldots, t_{N}}^{N} F=D_{t_{1}} D_{t_{2}} \ldots D_{t_{N}} F .
$$

For any real number $p \geq 1$ and any integer $N \geq 1$ we denote by $\mathbb{D}^{N, p}$ the completion of the set $\mathcal{S}$ with respect to the norm

$$
\|F\|_{N, p}=\left[E\left(|F|^{p}\right)+\sum_{i=1}^{N} E\left(\left\|D^{i} F\right\|_{L^{2}\left([0,1]^{i}\right)}^{p}\right)\right]^{\frac{1}{p}} .
$$

The domain of the derivative operator $D$ is the space $\mathbb{D}^{1,2}$.

The divergence operator $\delta$ is the adjoint of the derivative operator. The domain of the operator $\delta$, denoted by $\operatorname{Dom} \delta$, is the set of processes $u \in L^{2}([0,1] \times \Omega)$ such that there exists a square integrable random variable $\delta(u)$ verifying

$$
E(F \delta(u))=E\left(\int_{0}^{1} D_{t} F u_{t} d t\right),
$$

for any $F \in \mathcal{S}$. The operator $\delta$ is an extension of Itô's stochastic integral and we will make use of the notation $\delta(u)=\int_{0}^{1} u_{s} d W_{s}$.

We will recall some useful results from [14]. We refer the reader to this paper for their proof and a detailed account of these results. We also refer to $[\mathbf{1 6}],[\mathbf{1 5}]$ for any other property about operators $D$ and $\delta$. 
Proposition 1.1. Let $Y$ be a random variable in the space $\mathbb{D}^{1,2}$ such that $\int_{a}^{b}\left(D_{s} Y\right)^{2} d s>0$ a.s. for some $0 \leq a<b \leq 1$. Assume that $\left(D Y / \int_{a}^{b}\left(D_{s} Y\right)^{2} d s\right) I_{[a, b]}$ belongs to $\operatorname{Dom} \delta$. Then $Y$ has an absolutely continuous distribution with density $p$ that satisfies the inequality

$$
p(x) \leq E\left|\int_{a}^{b}\left(\frac{D_{s} Y}{\int_{a}^{b}\left(D_{s} Y\right)^{2} d s}\right) d W_{s}\right| .
$$

Proof: It follows from Proposition 1 and (2.6) in [14].

The following proposition is also a slight modification of Corollary 2 of $[\mathbf{1 4}]$.

Proposition 1.2. Let $Y$ be a random variable in the space $\mathbb{D}^{1,2}$ such that $\int_{0}^{1}\left(D_{s} Y\right)^{2} d s>0$ a.s. Let $Z$ be a positive square integrable random variable such that $\left(Z D Y / \int_{0}^{1}\left(D_{s} Y\right)^{2} d s\right) I_{[0,1]}$ belongs to $\operatorname{Dom} \delta$. Then, for any $f \in L^{2}(\mathbb{R})$, we have

$$
\left|E\left(f(Y)^{2} Z\right)\right|=\|f\|_{2}^{2} E\left|\delta\left(\frac{Z D Y}{\|D Y\|_{2}^{2}}\right)\right| .
$$

Proof: See Corollary 2 in [14]. The same proof works using a dominated convergence argument.

Lemma 1.3. Fix $p \geq 1$. Suppose that $u$ satisfies hypotheses (H1) and (H2). Let $Z \in \mathbb{D}^{1,2 p}$. Then, we have, for $0 \leq a<b \leq 1$ :

$$
\begin{aligned}
& E\left|\int_{a}^{b} Z \frac{D_{t} X_{b}}{\int_{a}^{b}\left(D_{t} X_{b}\right)^{2} d t} d W_{t}\right|^{p} \\
& \quad \leq C_{0}(b-a)^{-p / 2}\left(\left(E|Z|^{2 p}\right)^{1 / 2}+\left(E\left|\int_{a}^{b}\left(D_{t} Z\right)^{2} d t\right|^{p}\right)^{1 / 2}\right),
\end{aligned}
$$

where $C_{0}$ is a constant does not depend on $Z$.

Proof: See Lemma 10 in [14]. 


\section{Stochastic integration with respect to local time of the martingale}

Following the ideas of Eisenbaum [8], we consider first the space of functions for whose elements we can define a stochastic integration with respect to local time.

Let $f$ be a measurable function from $\mathbb{R} \times[0,1]$ into $\mathbb{R}$. We define the norm $\|\cdot\|$ by

$$
\|f\|=\left(\int_{0}^{1} \int_{\mathbb{R}} f^{2}(x, s) \frac{1}{s^{\frac{3}{4}}} d x d s\right)^{\frac{1}{2}} .
$$

Consider the set of functions

$$
\mathcal{H}=\{f:\|f\|<+\infty\}
$$

Since $\mathcal{H}$ is $L^{2}\left([0,1] \times \mathbb{R}, s^{-3 / 4} d x d s\right)$, it is a Banach space, in fact, it is a Hilbert space.

Let us consider $X$ a nondegenerate martingale of the type $X_{t}=$ $\int_{0}^{t} u_{s} d W_{s}$ where $u$ is an adapted stochastic process satisfying hypotheses (H1) and (H2). Let us show now how to define a stochastic integration over the plane with respect to the local time $L$ of the process $X$ for the elements of $\mathcal{H}$.

Let $f_{\Delta}$ be an elementary function,

$$
f_{\Delta}(x, s):=\sum_{\left(x_{k}, s_{l}\right) \in \Delta} f_{k l} I_{\left(x_{k}, x_{k+1}\right]}(x) I_{\left(s_{l}, s_{l+1}\right]}(s),
$$

where $\left(x_{k}\right)_{1 \leq k \leq m_{1}}$ is a finite sequence of real numbers, $\left(s_{l}\right)_{1 \leq l \leq m_{2}}$ is a subdivision of $[0,1],\left(f_{k l}\right)_{1 \leq k \leq m_{1} ; 1 \leq l \leq m_{2}}$ is a sequence of real numbers and finally, $\Delta=\left\{\left(x_{k}, s_{l}\right), 1 \leq k \leq m_{1}, 1 \leq l \leq m_{2}\right\}$. It is easy to check that the elementary functions are dense in $\mathcal{H}$.

We define the integration for the elementary function $f_{\Delta}$ with respect to the local time $L$ of the martingale $X$ as follows

$$
\int_{0}^{1} \int_{\mathbb{R}} f_{\Delta}(x, s) d L_{s}^{x}=\sum_{\left(x_{k}, s_{l}\right) \in \Delta} f_{k l}\left(L_{s_{l+1}}^{x_{k+1}}-L_{s_{l}}^{x_{k+1}}-L_{s_{l+1}}^{x_{k}}+L_{s_{l}}^{x_{k}}\right) .
$$


Let $f$ be a function of $\mathcal{H}$. Let us consider $\left(f_{n}\right)_{n \in \mathbb{N}}$ a sequence of elementary functions converging to $f$ in $\mathcal{H}$. We will check that the sequence $\left(\int_{0}^{1} \int_{\mathbb{R}} f_{n}(x, s) d L_{s}^{x}\right)_{n \in \mathbb{N}}$ converges in $L^{1}$ and that the limit does not depend on the choice of the sequence $\left(f_{n}\right)_{n \in \mathbb{N}}$. So, we will use this limit as the definition of the integral $\int_{0}^{1} \int_{\mathbb{R}} f(x, s) d L_{s}^{x}$.

First of all, let us see two previous lemmas.

Lemma 2.1. For any locally bounded Borel measurable function $f$ and any $t \in(0,1]$ we have

$$
\int_{\mathbb{R}} f(a) d_{a} L_{t}^{a}=-[f(X), X]_{t},
$$

where $d_{a} L_{t}^{a}$ denotes the integral with respect to $a \rightarrow L_{t}^{a}$.

Proof: It follows easily from Theorem 0.3 and Theorem 0.1 .

Lemma 2.2. Consider $f_{1}(x):=I_{(a, b]}(x)$ and $f_{2}(x):=I_{(c, d]}(x)$, where $a<b$ and $c<d$ are real numbers. Then for all $t_{i}<t_{j} \leq t$,

$$
\begin{aligned}
& E\left[f_{1}\left(X_{t_{i+1}}\right) f_{2}\left(X_{t_{j+1}}\right)\left(X_{t_{i+1}}-X_{t_{i}}\right)\left(X_{t_{j+1}}-X_{t_{j}}\right)\right] \\
\leq & E\left[f_{1}\left(X_{t_{i+1}}\right) f_{2}\left(X_{t_{j+1}}\right) C_{i j}\right],
\end{aligned}
$$

where

$$
\left\|C_{i j}\right\|_{2} \leq C \frac{\left(t_{i+1}-t_{i}\right)\left(t_{j+1}-t_{j}\right)}{\sqrt{t_{i+1}\left(t_{j+1}-t_{i+1}\right)}}
$$

and $C$ does not depend on $f_{1}, f_{2}, i$ and $j$.

Proof: When $f_{1}=f_{2}=f \in \mathcal{C}_{K}^{\infty}(\mathbb{R})$, this inequality is checked in the proof of Proposition 14 in [14]. The same proof also works when $f_{1} \neq f_{2}$ with $f_{1}, f_{2} \in \mathcal{C}_{K}^{\infty}(\mathbb{R})$. Now, fixed our functions $f_{1}, f_{2}$ let us consider sequences $f_{1}^{n} \uparrow f_{1}$ and $f_{2}^{n} \uparrow f_{2}$ with $f_{n}^{i} \in \mathcal{C}_{K}^{\infty}(\mathbb{R})$ for all $n$ and $i \in$ $\{1,2\}$. Then, the result can be obtained by a dominated convergence argument.

Theorem 2.3. Let $f$ be a function of $\mathcal{H}$. Then, there exists the integral $\int_{0}^{t} \int_{\mathbb{R}} f(x, s) d L_{s}^{x}$ for any $t \in[0,1]$. 
Proof: Let $f_{\Delta}$ be an elementary function. From Theorem 0.2 and Lemma 2.1 it is easy to get that the quadratic covariation $[f(X, .), X]_{t}$ exists and that

$$
\int_{0}^{t} \int_{\mathbb{R}} f_{\Delta}(x, s) d L_{s}^{x}=-\left[f_{\Delta}(X, \cdot), X\right]_{t}
$$

The key of the proof is to check that for all elementary function $f_{\Delta}$

$$
E\left(\left|\int_{0}^{t} \int_{\mathbb{R}} f_{\Delta}(x, s) d L_{s}^{x}\right|\right) \leq C\left\|f_{\Delta}\right\|,
$$

where the constant does not depend on $f_{\Delta}$.

Notice that,

$$
\begin{aligned}
& E\left(\left|\int_{0}^{t} \int_{\mathbb{R}} f_{\Delta}(x, s) d L_{s}^{x}\right|\right) \\
& =E\left(\left|\left[f_{\Delta}(X, \cdot), X\right]_{t}\right|\right) \\
& =E\left(\left|\lim _{n \rightarrow \infty} \sum_{t_{i} \in D_{n}, t_{i} \leq t}\left(f_{\Delta}\left(X_{t_{i+1}}, t_{i+1}\right)-f_{\Delta}\left(X_{t_{i}}, t_{i}\right)\right)\left(X_{t_{i+1}}-X_{t_{i}}\right)\right|\right) \\
& \text { (3) } \leq\left\{2 \liminf _{n \rightarrow \infty} E\left(\left|\sum_{t_{i} \in D_{n}, t_{i} \leq t} f_{\Delta}\left(X_{t_{i+1}}, t_{i+1}\right)\left(X_{t_{i+1}}-X_{t_{i}}\right)\right|^{2}\right)\right. \\
& \left.+2 \liminf _{n \rightarrow \infty} E\left(\left|\sum_{t_{i} \in D_{n}, t_{i} \leq t} f_{\Delta}\left(X_{t_{i}}, t_{i}\right)\left(X_{t_{i+1}}-X_{t_{i}}\right)\right|^{2}\right)\right\}^{\frac{1}{2}} \\
& :=\left(2\left(\liminf _{n \rightarrow \infty} I_{1}\right)+2\left(\liminf _{n \rightarrow \infty} I_{2}\right)\right)^{\frac{1}{2}}
\end{aligned}
$$

where in the last inequality we have used Fatou's lemma.

Along the study of $I_{1}$ and $I_{2}$ we will make use of the methods presented in the proofs of Propositions 13 and 14 in [14]. For the sake of completeness, we will give the main steps of our proofs in the study of $I_{2}$. 
By the isometry, and using Proposition 1.2 and Lemma 1.3 with $Z=$ $\int_{t_{i}}^{t_{i+1}} u_{s}^{2} d s, b=t_{i}$ and $a=0$

$$
\begin{aligned}
& I_{2}=E\left(\sum_{t_{i} \in D_{n}, t_{i} \leq t} f_{\Delta}^{2}\left(X_{t_{i}}, t_{i}\right) \int_{t_{i}}^{t_{i+1}} u_{s}^{2} d s\right) \\
& =\sum_{t_{i} \in D_{n}, t_{i} \leq t} E\left(f_{\Delta}^{2}\left(X_{t_{i}}, t_{i}\right) \int_{t_{i}}^{t_{i+1}} u_{s}^{2} d s\right) \\
& \leq \sum_{t_{i} \in D_{n}, t_{i} \leq t} \int_{\mathbb{R}} f_{\Delta}^{2}\left(x, t_{i}\right) d x E\left|\delta\left(\frac{\left(\int_{t_{i}}^{t_{i+1}} u_{s}^{2} d s\right) D X_{t_{i}}}{\left\|D X_{t_{i}}\right\|_{2}^{2}}\right)\right| \\
& \leq C \sum_{t_{i} \in D_{n}, t_{i} \leq t} \int_{\mathbb{R}} f_{\Delta}^{2}\left(x, t_{i}\right) d x t_{i}^{-\frac{1}{2}} \\
& \times\left(\sqrt{E\left|\int_{t_{i}}^{t_{i+1}} u_{s}^{2} d s\right|^{2}}+\sqrt{E \int_{0}^{t_{i}}\left(D_{t}\left(\int_{t_{i}}^{t_{i+1}} u_{s}^{2} d s\right)\right)^{2}} d t\right) \\
& \leq C \sum_{t_{i} \in D_{n}, t_{i} \leq t} \int_{\mathbb{R}} \sum_{k=1}^{m_{1}} \sum_{l=1}^{m_{2}} f_{k l}^{2} I_{\left(x_{k}, x_{k+1}\right]}(x) I_{\left(s_{l}, s_{l+1}\right]}\left(t_{i}\right) t_{i}^{-\frac{1}{2}}\left(t_{i+1}-t_{i}\right) d x \\
& =C \int_{\mathbb{R}} \sum_{k=1}^{m_{1}} \sum_{l=1}^{m_{2}} f_{k l}^{2} I_{\left(x_{k}, x_{k+1}\right]}(x)\left(\sum_{t_{i} \in D_{n}, t_{i} \leq t} I_{\left(s_{l}, s_{l+1}\right]}\left(t_{i}\right) t_{i}^{-\frac{1}{2}}\left(t_{i+1}-t_{i}\right)\right) d x \\
& =C \int_{\mathbb{R}} \sum_{k=1}^{m_{1}} \sum_{l=1}^{m_{2}} f_{k l}^{2} I_{\left(x_{k}, x_{k+1}\right]}(x) \\
& \times\left(\int_{0}^{t} \sum_{t_{i} \in D_{n}, t_{i} \leq t} I_{\left(s_{l}, s_{l+1}\right]}\left(t_{i}\right) t_{i}^{-\frac{1}{2}} I_{\left(t_{i}, t_{i+1}\right]}(s) d s\right) d x .
\end{aligned}
$$

Using the condition (M) over the partitions, we have that, by bounded convergence,

$$
\lim _{n \rightarrow \infty} \int_{0}^{t} \sum_{t_{i} \in D_{n}, t_{i} \leq t} I_{\left(s_{l}, s_{l+1}\right]}\left(t_{i}\right) t_{i}^{-\frac{1}{2}} I_{\left(t_{i}, t_{i+1}\right]}(s) d s=\int_{0}^{t} I_{\left(s_{l}, s_{l+1}\right]}(s) s^{-\frac{1}{2}} d s,
$$


and then,

$$
\liminf _{n \rightarrow \infty} I_{2} \leq C \int_{\mathbb{R}} \sum_{k=1}^{m_{1}} \sum_{l=1}^{m_{2}} f_{k l}^{2} I_{\left(x_{k}, x_{k+1}\right]}(x) \int_{0}^{t} I_{\left(s_{l}, s_{l+1}\right]}(s) s^{-\frac{1}{2}} d s d x
$$

$$
\begin{aligned}
& =C \int_{0}^{t} \int_{\mathbb{R}} f_{\Delta}^{2}(x, s) \frac{1}{\sqrt{s}} d x d s \\
& \leq C\left\|f_{\Delta}\right\|^{2} .
\end{aligned}
$$

On the other hand,

$$
\begin{aligned}
I_{1} & =E\left(\left|\sum_{t_{i} \in D_{n}, t_{i} \leq t} f_{\Delta}\left(X_{t_{i+1}}, t_{i+1}\right)\left(X_{t_{i+1}}-X_{t_{i}}\right)\right|^{2}\right) \\
& =E\left(\sum_{t_{i} \in D_{n}, t_{i} \leq t} f_{\Delta}^{2}\left(X_{t_{i+1}}, t_{i+1}\right)\left(X_{t_{i+1}}-X_{t_{i}}\right)^{2}\right)
\end{aligned}
$$

(5)

$$
\begin{aligned}
& +2 E\left(\sum_{t_{i}, t_{j} \in D_{n}, t_{i}<t_{j} \leq t} f_{\Delta}\left(X_{t_{i+1}}, t_{i+1}\right) f_{\Delta}\left(X_{t_{j+1}}, t_{j+1}\right)\right. \\
& \left.\times\left(X_{t_{i+1}}-X_{t_{i}}\right)\left(X_{t_{j+1}}-X_{t_{j}}\right)\right) \\
& :=I_{1,1}+2 I_{1,2} .
\end{aligned}
$$

Following now the methods of Proposition 14 of [14] and using again Propositions 1.2 and 1.3 as we did in the study of $I_{2}$, we get that

$$
I_{1,1} \leq C \sum_{t_{i} \in D_{n}, t_{i} \leq t} \int_{\mathbb{R}} f_{\Delta}^{2}\left(x, t_{i}\right) d x t_{i+1}^{-\frac{1}{2}}\left(t_{i+1}-t_{i}\right) .
$$

By similar computations to those of the term $I_{2}$ we obtain that

$$
\liminf _{n \rightarrow \infty} I_{1,1} \leq C\left\|f_{\Delta}\right\|^{2}
$$


Let us study now $I_{1,2}$. Using Lemma 2.2, notice that

$$
\begin{array}{r}
I_{1,2}=E\left(\sum_{t_{i}, t_{j} \in D_{n}, t_{i}<t_{j} \leq t} f_{\Delta}\left(X_{t_{i+1}}, t_{i+1}\right) f_{\Delta}\left(X_{t_{j+1}}, t_{j+1}\right)\right. \\
\left.\times\left(X_{t_{i+1}}-X_{t_{i}}\right)\left(X_{t_{j+1}}-X_{t_{j}}\right)\right) \\
\leq \sum_{t_{i}, t_{j} \in D_{n}, t_{i}<t_{j} \leq t} E\left(f_{\Delta}\left(X_{t_{i+1}}, t_{i+1}\right) f_{\Delta}\left(X_{t_{j+1}}, t_{j+1}\right) C_{i j}\right) .
\end{array}
$$

Following again the methods of the proof of Proposition 14 of [14] -more precisely, the proof of inequalities (5.36) and (5.37) - the last expression is bounded by

$$
\begin{aligned}
& \sum_{t_{i}, t_{j} \in D_{n}, t_{i}<t_{j} \leq t} E\left(f_{\Delta}^{2}\left(X_{t_{i+1}}, t_{i+1}\right) f_{\Delta}^{2}\left(X_{t_{j+1}}, t_{j+1}\right)\right)^{\frac{1}{2}} E\left(C_{i j}^{2}\right)^{\frac{1}{2}} \\
& \leq C \sum_{t_{i}, t_{j} \in D_{n}, t_{i}<t_{j} \leq t} \frac{\left(t_{i+1}-t_{i}\right)\left(t_{j+1}-t_{j}\right)}{\left(t_{i+1}\left(t_{j+1}-t_{i+1}\right)\right)^{\frac{3}{4}}} \\
& \times\left(\int_{\mathbb{R}} f_{\Delta}^{2}\left(x, t_{i+1}\right) d x\right)^{\frac{1}{2}}\left(\int_{\mathbb{R}} f_{\Delta}^{2}\left(x, t_{j+1}\right) d x\right)^{\frac{1}{2}} \\
& \leq \sum_{t_{i}, t_{j} \in D_{n}, t_{i}<t_{j} \leq t} \frac{\left(t_{i+1}-t_{i}\right)\left(t_{j+1}-t_{j}\right)}{\left(t_{i+1}\left(t_{j+1}-t_{i+1}\right)\right)^{\frac{3}{4}}} \\
& \times\left(\int_{\mathbb{R}} f_{\Delta}^{2}\left(x, t_{i+1}\right) d x+\int_{\mathbb{R}} f_{\Delta}^{2}\left(x, t_{j+1}\right) d x\right) \\
& :=C\left(I_{1,2,1}+I_{1,2,2}\right) .
\end{aligned}
$$

Since

$$
\begin{aligned}
& \sum_{t_{j} \in D_{n}, t_{i}<t_{j} \leq t} \frac{\left(t_{j+1}-t_{j}\right)}{\left(t_{j+1}-t_{i+1}\right)^{\frac{3}{4}}} \\
& \quad=\int_{t_{i+1}}^{t} \sum_{t_{j} \in D_{n}, t_{i}<t_{j} \leq t} \frac{1}{\left(t_{j+1}-t_{i+1}\right)^{\frac{3}{4}}} I_{\left(t_{j}, t_{j+1}\right]}(s) d s
\end{aligned}
$$


we get that

$$
\begin{aligned}
I_{1,2,1} & \leq \sum_{t_{i} \in D_{n}, t_{i}<t_{j} \leq t} \frac{\left(t_{i+1}-t_{i}\right)}{t_{i+1}^{\frac{3}{4}}} \int_{t_{i+1}}^{t} \frac{1}{\left(s-t_{i+1}\right)^{\frac{3}{4}}} d s\left(\int_{\mathbb{R}} f_{\Delta}^{2}\left(x, t_{i+1}\right) d x\right) \\
& \leq C \sum_{t_{i} \in D_{n}, t_{i}<t_{j} \leq t} \frac{\left(t_{i+1}-t_{i}\right)}{t_{i+1}^{\frac{3}{4}}} \int_{\mathbb{R}} f_{\Delta}^{2}\left(x, t_{i+1}\right) d x .
\end{aligned}
$$

And this clearly yields that

$$
\liminf _{n \rightarrow \infty} I_{1,2,1} \leq C \int_{0}^{t} \int_{\mathbb{R}} f_{\Delta}^{2}(x, s) \frac{1}{s^{\frac{3}{4}}} d s=C\left\|f_{\Delta}\right\|^{2} .
$$

Finally we have to consider $I_{1,2,2}$. First of all, notice that

$$
\begin{aligned}
I_{1,2,2}: & =\sum_{t_{i}, t_{j} \in D_{n}, t_{i}<t_{j} \leq t} \frac{\left(t_{i+1}-t_{i}\right)\left(t_{j+1}-t_{j}\right)}{\left(t_{i+1}\left(t_{j+1}-t_{i+1}\right)\right)^{\frac{3}{4}}}\left(\int_{\mathbb{R}} f_{\Delta}^{2}\left(x, t_{j+1}\right) d x\right) \\
= & \sum_{t_{i}, t_{j} \in D_{n}, t_{i}<t_{j} \leq t} \frac{\left(t_{i+1}-t_{i}\right)\left(t_{j+1}-t_{j}\right)}{\left(t_{i+1}\left(t_{j+1}-t_{i+1}\right)\right)^{\frac{3}{4}}} \\
& \times\left(\int_{\mathbb{R}} \sum_{k=1}^{m_{1}} \sum_{l=1}^{m_{2}} f_{k l}^{2} I_{\left(x_{k}, x_{k+1}\right]}(x) I_{\left(s_{l}, s_{l+1}\right]}\left(t_{j+1}\right) d x\right) \\
\leq & \int_{\mathbb{R}} \sum_{k=1}^{m_{1}} \sum_{l=1}^{m_{2}} f_{k l}^{2} I_{\left(x_{k}, x_{k+1}\right]}(x) \sum_{t_{i} \in D_{n}, t_{i}<t} \frac{\left(t_{i+1}-t_{i}\right)}{t_{i+1}^{\frac{3}{4}}} \\
& \times \sum_{t_{j} \in D_{n}, t_{i}<t_{j} \leq t} \int_{t_{i+1}}^{t} \frac{1}{\left(s-t_{i+1}\right)^{\frac{3}{4}}} I_{\left(s_{l}, s_{l+1}\right]}\left(t_{j+1}\right) I_{\left(t_{j}, t_{j+1}\right]}(s) d s d x .
\end{aligned}
$$

From the obvious inequality

$$
I_{\left(s_{l}, s_{l+1}\right]}\left(t_{j+1}\right) I_{\left(t_{j}, t_{j+1}\right]}(s) \leq I_{\left(s_{l}, s_{l+1}\right]}(s) I_{\left(t_{j}, t_{j+1}\right]}(s)+I_{\left(t_{j}, s_{l}\right]}(s) I_{\left(t_{j}, t_{j+1}\right]}\left(s_{l}\right),
$$

we obtain the bound

$$
I_{1,2,2} \leq I_{1,2,2,1}+I_{1,2,2,2}
$$


where

$$
\begin{aligned}
I_{1,2,2,1}= & \int_{\mathbb{R}} \sum_{k=1}^{m_{1}} \sum_{l=1}^{m_{2}} f_{k l}^{2} I_{\left(x_{k}, x_{k+1}\right]}(x) \sum_{t_{i} \in D_{n}, t_{i}<t} \frac{\left(t_{i+1}-t_{i}\right)}{t_{i+1}^{\frac{3}{4}}} \\
& \times \sum_{t_{j} \in D_{n}, t_{i}<t_{j} \leq t}^{t} \int_{t_{i+1}}^{t} \frac{1}{\left(s-t_{i+1}\right)^{\frac{3}{4}}} I_{\left(s_{l}, s_{l+1}\right]}(s) I_{\left(t_{j}, t_{j+1}\right]}(s) d s d x \\
I_{1,2,2,2}= & \int_{\mathbb{R}} \sum_{k=1}^{m_{1}} \sum_{l=1}^{m_{2}} f_{k l}^{2} I_{\left(x_{k}, x_{k+1}\right]}(x) \sum_{t_{i} \in D_{n}, t_{i}<t} \frac{\left(t_{i+1}-t_{i}\right)}{t_{i+1}^{\frac{3}{4}}} \\
& \times \sum_{t_{j} \in D_{n}, t_{i}<t_{j} \leq t} \int_{t_{i+1}}^{t} \frac{1}{\left(s-t_{i+1}\right)^{\frac{3}{4}}} I_{\left(t_{j}, s_{l}\right]}(s) I_{\left(t_{j}, t_{j+1}\right]}\left(s_{l}\right) d s d x .
\end{aligned}
$$

Now, since we can write

$$
I_{1,2,2,1}=\int_{\mathbb{R}_{t_{i}} \in D_{n}, t_{i}<t} \frac{\left(t_{i+1}-t_{i}\right)}{t_{i+1}^{\frac{3}{4}}} \int_{t_{i+1}}^{t} f_{\Delta}^{2}(x, s) \frac{1}{\left(s-t_{i+1}\right)^{\frac{3}{4}}} d s d x
$$

using an argument of bounded convergence we have that

$$
\begin{aligned}
\liminf _{n \rightarrow \infty} I_{1,2,2,1} & \leq \int_{\mathbb{R}} \int_{0}^{t} \frac{1}{u^{\frac{3}{4}}} \int_{u}^{t} f_{\Delta}^{2}(x, s) \frac{1}{(s-u)^{\frac{3}{4}}} d s d u d x \\
& =\int_{\mathbb{R}} \int_{0}^{t} f_{\Delta}^{2}(x, s) \int_{0}^{s} \frac{1}{u^{\frac{3}{4}}} \frac{1}{(s-u)^{\frac{3}{4}}} d u d s d x \\
& \leq C \int_{\mathbb{R}} \int_{0}^{t} f_{\Delta}^{2}(x, s) \frac{1}{s^{\frac{1}{2}}} d s d x \\
& \leq C\left\|f_{\Delta}\right\|^{2} .
\end{aligned}
$$

On the other hand, observe that fixed $l$, there exists only one $j$ (that we will denote by $j(l))$ such that $t_{j(l)}<s_{l} \leq t_{j(l)+1}$. So,

$$
\begin{aligned}
& \sum_{t_{i}, t_{j} \in D_{n}, t_{i}<t_{j} \leq t} \frac{\left(t_{i+1}-t_{i}\right)}{t_{i+1}^{\frac{3}{4}}} \int_{t_{i+1}}^{t} \frac{1}{\left(s-t_{i+1}\right)^{\frac{3}{4}}} I_{\left(t_{j}, s_{l}\right]}(s) I_{\left(t_{j}, t_{j+1}\right]}\left(s_{l}\right) d s \\
& \leq \sum_{t_{i} \in D_{n}, t_{i}<t_{j(l)} \leq t} \frac{\left(t_{i+1}-t_{i}\right)}{t_{i+1}^{\frac{3}{4}}} \int_{t_{j(l)}}^{t_{j(l)+1}} \frac{1}{\left(s-t_{i+1}\right)^{\frac{3}{4}}} d s .
\end{aligned}
$$


Now, using that for $i<j(l)$

$$
\int_{t_{j(l)}}^{t_{j(l)+1}} \frac{1}{\left(s-t_{i+1}\right)^{\frac{3}{4}}} d s \leq \int_{t_{j(l)}}^{t_{j(l)+1}} \frac{1}{\left(s-t_{j(l)}\right)^{\frac{3}{4}}} d s \leq 4\left|D_{n}\right|^{\frac{1}{4}},
$$

and that

we obtain easily that

$$
\sum_{t_{i} \in D_{n}, t_{i}<t_{j(l)} \leq t} \frac{\left(t_{i+1}-t_{i}\right)}{t_{i+1}^{\frac{3}{4}}} \leq \int_{0}^{1} \frac{1}{s^{\frac{3}{4}}} d s<\infty,
$$

$$
\lim _{n \rightarrow \infty} I_{1,2,2,2}=0 .
$$

So, putting together (3)-(11), we have proved (2).

Now, given $f \in \mathcal{H}$, let us consider $\left\{f_{n}\right\}_{n \in \mathbb{N}}$ a sequence of elementary functions converging to $f$ in $\mathcal{H}$, and we define

$$
\int_{0}^{t} \int_{\mathbb{R}} f(x, s) d L_{s}^{x}=L^{1}-\lim _{n \rightarrow \infty}\left(\int_{0}^{t} \int_{\mathbb{R}} f_{n}(x, s) d L_{s}^{x}\right) .
$$

Clearly, this limit exists. Indeed, for any $\varepsilon>0$ there exists $n_{0}$ such that for any $n, m \geq n_{0},\left\|f_{n}-f_{m}\right\|<\varepsilon$ and using inequality (2) we obtain that

$$
\begin{aligned}
& E\left|\int_{0}^{t} \int_{\mathbb{R}} f_{n}(x, s) d L_{s}^{x}-\int_{0}^{t} \int_{\mathbb{R}} f_{m}(x, s) d L_{s}^{x}\right| \\
= & E\left|\int_{0}^{t} \int_{\mathbb{R}}\left(f_{n}(x, s)-f_{m}(x, s)\right) d L_{s}^{x}\right| \leq\left\|f_{n}-f_{m}\right\|<\varepsilon .
\end{aligned}
$$

Moreover, using again inequality (2), it is clear that the definition does not depend on the choice of the sequence $\left(f_{n}\right)$. Indeed, given $\left(f_{n}^{1}\right)_{n \in \mathbb{N}}$ and $\left(f_{n}^{2}\right)_{n \in \mathbb{N}}$ two sequences converging to $f$ in $\mathcal{H}$, we have

$$
\begin{aligned}
E\left(\left|\int_{0}^{t} \int_{\mathbb{R}} f_{n}^{1}(x, s) d L_{s}^{x}-\int_{0}^{t} \int_{\mathbb{R}} f_{n}^{2}(x, s) d L_{s}^{x}\right|\right) & \\
\leq\left\|f_{n}^{1}-f_{n}^{2}\right\| & \leq\left\|f_{n}^{1}-f\right\|+\left\|f-f_{n}^{2}\right\|,
\end{aligned}
$$

that goes to zero when $n$ tends to infinity.

Remark 2.4. If $f$ satisfies condition (C), from Theorem 0.2 we know that the quadratic covariation $[f(X, \cdot), X]$ exists. Moreover, if $f \in \mathcal{H}$, from the uniqueness of the extension in the construction of the integral in Theorem 2.3 we get that

$$
\int_{0}^{t} \int_{\mathbb{R}} f(x, s) d L_{s}^{x}=-[f(X, \cdot), X]_{t} .
$$


The following result is an obvious consequence of Theorem 0.2 and Remark 2.4.

Corollary 2.5. Let $u$ be a process satisfying (H1) and (H2). Set $X=$ $\int_{0}^{t} u_{s} d W_{s}$. Consider a sequence $D_{n}$ of partitions of $[0,1]$ verifying conditions $(M)$. Let $F(x, t)$ be an absolutely continuous function in $x$ such that the partial derivative $f(\cdot, t)$ satisfies $(C)$. Then, if $f \in \mathcal{H}$, we have the following extension for the Itô's formula:

$F\left(X_{t}, t\right)=F(0,0)+\int_{0}^{t} f\left(X_{s}, s\right) d X_{s}-\frac{1}{2} \int_{0}^{t} \int_{\mathbb{R}} f(x, s) d L_{s}^{x}+\int_{0}^{t} F\left(X_{s}, d s\right)$.

\section{Itô's formula extension}

Now we can state the main result of this paper.

\section{Theorem 3.1.}

Hypothesis over the martingale:

1) Let $u$ be an adapted process satisfying (H1) and (H2). Set $X_{t}=$ $\int_{0}^{t} u_{s} d W_{s}$.

Hypothesis over the function:

1) Let $F$ be a function defined on $\mathbb{R} \times[0,1]$ such that $F$ admits first order Radon-Nikodym derivatives with respect to each parameter. Assume that these derivatives are measurable in both variables.

2) Assume also that these derivatives satisfy that for every $A \in \mathbb{R}$,

$$
\begin{gathered}
\int_{0}^{1} \int_{-A}^{A}\left|\frac{\partial F}{\partial t}(x, s)\right| d x \frac{1}{\sqrt{s}} d s<+\infty \\
\int_{0}^{1} \int_{-A}^{A}\left(\frac{\partial F}{\partial x}(x, s)\right)^{2} d x \frac{1}{\sqrt{s}} d s<+\infty .
\end{gathered}
$$

Then, for all $t \in[0,1]$,

$$
\begin{aligned}
F\left(X_{t}, t\right)=F(0,0)+\int_{0}^{t} \frac{\partial F}{\partial x}\left(X_{s}, s\right) d X_{s}+ & \int_{0}^{t} \frac{\partial F}{\partial t}\left(X_{s}, s\right) d s \\
& -\frac{1}{2} \int_{0}^{t} \int_{\mathbb{R}} \frac{\partial F}{\partial x}(x, s) d L_{s}^{x} .
\end{aligned}
$$

Remark 3.2. Notice that under the hypotheses of Theorem 3.1, it is possible that $\frac{\partial F}{\partial x}$ does not belong to the space $\mathcal{H}$. In this case, using the localization arguments, we can always assume that $\left(\frac{\partial F}{\partial x}(x, s) I_{(\varepsilon, t)}(s), x \in \mathbb{R}\right.$, 
$s \in[0,1])$ belongs to $\mathcal{H}$ for any $\varepsilon>0$ and we define, in the previous theorem,

$$
\int_{0}^{t} \int_{\mathbb{R}} \frac{\partial F}{\partial x}(x, s) d L_{s}^{x}=\lim _{\varepsilon \rightarrow 0} \int_{0}^{1} \int_{\mathbb{R}} \frac{\partial F}{\partial x}(x, s) I_{(\varepsilon, t)}(s) d L_{s}^{x} .
$$

At the end of the proof, we will justify that this limit exists.

Proof: Using localization arguments we can assume that $F$ has compact support and

$$
\begin{gathered}
\int_{0}^{1} \int_{\mathbb{R}}\left|\frac{\partial F}{\partial t}(x, s)\right| d x \frac{1}{\sqrt{s}} d s<+\infty \\
\int_{0}^{1} \int_{\mathbb{R}}\left(\frac{\partial F}{\partial x}(x, s)\right)^{2} d x \frac{1}{\sqrt{s}} d s<+\infty .
\end{gathered}
$$

Let $g \in \mathcal{C}^{\infty}$ be a function with compact support from $\mathbb{R}$ to $\mathbb{R}^{+}$such that $\int_{\mathbb{R}} g(s) d s=1$. We define, for any $n \in \mathbb{N}$,

$$
g_{n}(s)=n g(n s)
$$

and

$$
F_{n}(x, t)=\int_{0}^{1} \int_{\mathbb{R}} F(y, s) g_{n}(t-s) g_{n}(x-y) d y d s .
$$

Then $F_{n} \in \mathcal{C}^{\infty}(\mathbb{R} \times[0,1])$. Hence, by the usual Itô's formula, for every $\varepsilon>0$, we can write

$$
\begin{array}{r}
F_{n}\left(X_{t}, t\right)=F_{n}\left(X_{\varepsilon}, \varepsilon\right)+\int_{\varepsilon}^{t} \frac{\partial F_{n}}{\partial x}\left(X_{s}, s\right) d X_{s}+\int_{\varepsilon}^{t} \frac{\partial F_{n}}{\partial t}\left(X_{s}, s\right) d s \\
+\frac{1}{2} \int_{\varepsilon}^{t} u_{s}^{2} \frac{\partial^{2} F_{n}}{\partial x^{2}}\left(X_{s}, s\right) d s
\end{array}
$$

Using the arguments of Azéma et al. [1] we will study the convergence of (12).

Since $F$ is a continuous function with compact support, it is easy to check that $\left(F_{n}\left(X_{t}, t\right)\right)_{n \in \mathbb{N}}$ converges in probability to $F\left(X_{t}, t\right)$.

On the other hand

$$
\int_{0}^{1} \int_{\mathbb{R}}\left|\frac{\partial F}{\partial t}(x, s)\right| d x d s \leq \int_{0}^{1} \int_{\mathbb{R}}\left|\frac{\partial F}{\partial t}(x, s)\right| d x \frac{1}{\sqrt{s}} d s<+\infty .
$$

Hence, $\frac{\partial F}{\partial t} \in L^{1}(\mathbb{R} \times[0,1])$. Under our hypothesis over the martingale $X$, it follows from Proposition 1.1 and Lemma 1.3 that for any $t \in[0,1]$, the 
random variable $X_{t}$ is absolutely continuous with density $p_{t}$ satisfying the estimate

$$
p_{t}(x) \leq \frac{C}{\sqrt{t}}
$$

Then, it is easy to see that $\left(\int_{\varepsilon}^{t} \frac{\partial F_{n}}{\partial t}\left(X_{s}, s\right) d s\right)_{n \in \mathbb{N}}$ converges in probability to $\left(\int_{\varepsilon}^{t} \frac{\partial F}{\partial t}\left(X_{s}, s\right) d s\right)$. Indeed,

$$
\begin{aligned}
& E\left(\left|\int_{\varepsilon}^{t}\left(\frac{\partial F_{n}}{\partial t}\left(X_{s}, s\right)-\frac{\partial F}{\partial t}\left(X_{s}, s\right)\right) d s\right|\right) \\
& \quad \leq \int_{\varepsilon}^{t} \int_{\mathbb{R}}\left|\frac{\partial F_{n}}{\partial t}(x, s)-\frac{\partial F}{\partial t}(x, s)\right| p_{s}(x) d x d s \\
& \quad \leq C \int_{\varepsilon}^{t} \int_{\mathbb{R}}\left|\frac{\partial F_{n}}{\partial t}(x, s)-\frac{\partial F}{\partial t}(x, s)\right| \frac{1}{\sqrt{s}} d x d s \\
& \quad \leq \frac{C}{\sqrt{\varepsilon}} \int_{\varepsilon}^{t} \int_{\mathbb{R}}\left|\frac{\partial F_{n}}{\partial t}(x, s)-\frac{\partial F}{\partial t}(x, s)\right| d x d s
\end{aligned}
$$

that goes to zero, when $n$ tends to infinity, since $\frac{\partial F}{\partial t} \in L^{1}(\mathbb{R} \times[0,1])$ and

$$
\frac{\partial F_{n}}{\partial t}(x, t)=\int_{0}^{1} \int_{\mathbb{R}} \frac{\partial F}{\partial t}(y, s) g_{n}(t-s) g_{n}(x-y) d y d s .
$$

Similarly, we can prove that $\left(\int_{\varepsilon}^{t} \frac{\partial F_{n}}{\partial x}\left(X_{s}, s\right) d X_{s}\right)_{n \in \mathbb{N}}$ converges in probability to $\left(\int_{\varepsilon}^{t} \frac{\partial F}{\partial x}\left(X_{s}, s\right) d X_{s}\right)$. Indeed, using the same arguments we get that $\frac{\partial F}{\partial x} \in L^{2}(\mathbb{R} \times[0,1])$. Then,

$$
\begin{gathered}
E\left(\left|\int_{\varepsilon}^{t}\left(\frac{\partial F_{n}}{\partial x}\left(X_{s}, s\right)-\frac{\partial F}{\partial x}\left(X_{s}, s\right)\right) d X_{s}\right|^{2}\right) \\
\quad=E\left(\left|\int_{\varepsilon}^{t}\left(\frac{\partial F_{n}}{\partial x}\left(X_{s}, s\right)-\frac{\partial F}{\partial x}\left(X_{s}, s\right)\right) u_{s} d W_{s}\right|^{2}\right) \\
=E\left(\int_{\varepsilon}^{t}\left(\frac{\partial F_{n}}{\partial x}\left(X_{s}, s\right)-\frac{\partial F}{\partial x}\left(X_{s}, s\right)\right)^{2} u_{s}^{2} d s\right) .
\end{gathered}
$$


Following the same ideas of Proposition 12 in [14], Proposition 1.2 and Lemma 1.3 yield the following bound for the last expression

(13) $C \int_{\varepsilon}^{t} \int_{\mathbb{R}}\left(\frac{\partial F_{n}}{\partial x}(x, s)-\frac{\partial F}{\partial x}(x, s)\right)^{2} \frac{1}{\sqrt{s}} d x d s$

$$
\leq \frac{C}{\sqrt{\varepsilon}} \int_{\varepsilon}^{t} \int_{\mathbb{R}}\left(\frac{\partial F_{n}}{\partial x}(x, s)-\frac{\partial F}{\partial x}(x, s)\right)^{2} d x d s
$$

that goes to zero when $n$ tends to infinity, since $\frac{\partial F}{\partial x} \in L^{2}(\mathbb{R} \times[0,1])$ and

$$
\frac{\partial F_{n}}{\partial x}(x, t)=\int_{0}^{1} \int_{\mathbb{R}} \frac{\partial F}{\partial x}(y, s) g_{n}(t-s) g_{n}(x-y) d y d s .
$$

So, letting $n$ to infinity in (12), we get that the sequence

$$
\left(\frac{1}{2} \int_{\varepsilon}^{t} u_{s}^{2} \frac{\partial^{2} F_{n}}{\partial x^{2}}\left(X_{s}, s\right) d s\right)_{n \in \mathbb{N}}
$$

converges in probability to

$$
F\left(X_{t}, t\right)-F\left(X_{\varepsilon}, \varepsilon\right)-\int_{\varepsilon}^{t} \frac{\partial F}{\partial x}\left(X_{s}, s\right) d X_{s}-\int_{\varepsilon}^{t} \frac{\partial F}{\partial t}\left(X_{s}, s\right) d s .
$$

But, since $\frac{\partial F_{n}}{\partial x}(x, s) I_{(\varepsilon, t)}(s) \in \mathcal{H}$, from Theorem 0.2 and Corollary 2.5, we get that

$$
\begin{aligned}
\int_{\varepsilon}^{t} u_{s}^{2} \frac{\partial^{2} F_{n}}{\partial x^{2}}\left(X_{s}, s\right) d s & =\left[\frac{\partial F_{n}}{\partial x}(X, \cdot), X\right]_{t}-\left[\frac{\partial F_{n}}{\partial x}(X, \cdot), X\right]_{\varepsilon} \\
& =-\int_{0}^{1} \int_{\mathbb{R}} \frac{\partial F_{n}}{\partial x}(x, s) I_{(\varepsilon, t)}(s) d L_{s}^{x}
\end{aligned}
$$

The next step of the proof is to check that $\left(\frac{\partial F_{n}}{\partial x}(x, s) I_{(\varepsilon, t)}(s), x \in \mathbb{R}\right.$, $s \in[0,1])_{n \in \mathbb{N}}$ converges in $\mathcal{H}$ to $\left(\frac{\partial F}{\partial x}(x, s) I_{(\varepsilon, t)}(s), x \in \mathbb{R}, s \in[0,1]\right)$. It suffices to notice that,

$$
\begin{aligned}
\int_{\varepsilon}^{t} \int_{\mathbb{R}}\left(\frac{\partial F_{n}}{\partial x}(x, s)-\frac{\partial F}{\partial x}(x, s)\right)^{2} \frac{1}{s^{\frac{3}{4}}} d x d s & \\
& \leq \frac{1}{\varepsilon^{\frac{3}{4}}} \int_{\varepsilon}^{t} \int_{\mathbb{R}}\left(\frac{\partial F_{n}}{\partial x}(x, s)-\frac{\partial F}{\partial x}(x, s)\right)^{2} d x d s
\end{aligned}
$$


that converges to zero when $n$ tends to infinity. Then, we clearly have proved that

$$
\left(\int_{0}^{1} \int_{\mathbb{R}} \frac{\partial F_{n}}{\partial x}(x, s) I_{(\varepsilon, t)}(s) d L_{s}^{x}\right)_{n \in \mathbb{N}}
$$

converges in $L^{1}$ to $\int_{0}^{1} \int_{\mathbb{R}} \frac{\partial F}{\partial x}(x, s) I_{(\varepsilon, t)}(s) d L_{s}^{x}$.

So, we have that for any $\varepsilon>0$

$$
\begin{aligned}
F\left(X_{t}, t\right)=F\left(X_{\varepsilon}, \varepsilon\right)+\int_{\varepsilon}^{t} \frac{\partial F}{\partial x} & \left(X_{s}, s\right) d X_{s}+\int_{\varepsilon}^{t} \frac{\partial F}{\partial t}\left(X_{s}, s\right) d s \\
& -\frac{1}{2} \int_{0}^{1} \int_{\mathbb{R}} \frac{\partial F}{\partial x}(x, s) I_{(\varepsilon, t)}(s) d L_{s}^{x}
\end{aligned}
$$

The last step is to let $\varepsilon$ to zero. But we need to check that the limit of the stochastic integral exists. Actually, it is enough to show that

$$
E\left(\left|\int_{0}^{t} \frac{\partial F}{\partial t}\left(X_{s}, s\right) d s\right|\right)<\infty
$$

and that

$$
E\left(\int_{0}^{t} \frac{\partial F}{\partial x}\left(X_{s}, s\right) d X_{s}\right)^{2}<\infty
$$

But,

$$
E\left(\left|\int_{0}^{t} \frac{\partial F}{\partial t}\left(X_{s}, s\right) d s\right|\right) \leq C \int_{0}^{1} \int_{\mathbb{R}}\left|\frac{\partial F}{\partial t}(x, s)\right| d x \frac{1}{\sqrt{s}} d s<+\infty .
$$

On the other hand, following the same type of arguments that in (13), we are able to write

$$
\begin{aligned}
E\left(\int_{0}^{t} \frac{\partial F}{\partial x}\left(X_{s}, s\right) d X_{s}\right)^{2} & =E\left(\int_{0}^{t}\left(\frac{\partial F}{\partial x}\left(X_{s}, s\right)\right)^{2} u_{s}^{2} d s\right) \\
& \leq C \int_{\mathbb{R}} \int_{0}^{t}\left(\frac{\partial F}{\partial x}(x, s)\right)^{2} \frac{1}{\sqrt{s}} d s d x<\infty .
\end{aligned}
$$

Letting $\varepsilon$ to zero, the proof is finished.

When $\frac{\partial F}{\partial x}$ does not belong to the space $\mathcal{H}$, as we explained in Remark 3.2 , we define

$$
\int_{0}^{t} \int_{\mathbb{R}} \frac{\partial F}{\partial x}(x, s) d L_{s}^{x}=\lim _{\varepsilon \rightarrow 0} \int_{0}^{1} \int_{\mathbb{R}} \frac{\partial F}{\partial x}(x, s) I_{(\varepsilon, t)}(s) d L_{s}^{x} .
$$

This limit clearly exists in probability since all the other limits in (14) exist. 


\section{Acknowledgements}

This work was partially supported by DGES Grants MTM2006-01351 (Carles Rovira) and MTM2006-06427 (Xavier Bardina).

\section{References}

[1] J. Azéma, T. Jeulin, F. Knight, And M. Yor, Quelques calculs de compensateurs impliquant l'injectivité de certains processus croissants, in: "Séminaire de Probabilités, XXXII", Lecture Notes in Math. 1686, Springer, Berlin, 1998, pp. 316-327.

[2] X. Bardina And M. Jolis, An extension of Itô's formula for elliptic diffusion processes, Stochastic Process. Appl. 69(1) (1997), $83-109$.

[3] X. Bardina And M. Jolis, Estimation of the density of hypoelliptic diffusion processes with application to an extended Itô's formula, J. Theoret. Probab. 15(1) (2002), 223-247.

[4] X. Bardina And C. Rovira, On Itô's formula for elliptic diffusion processes, Bernoulli 13(3) (2007), 820-830.

[5] N. Bouleau ANd M. Yor, Sur la variation quadratique des temps locaux de certaines semimartingales, C. R. Acad. Sci. Paris Sér. I Math. 292(9) (1981), 491-494.

[6] G. Di Nunno, T. Meyer-Brandis, B. Øksendal, and F. Proske, Malliavin calculus and anticipative Itô formulae for Lévy processes, Infin. Dimens. Anal. Quantum Probab. Relat. Top. 8(2) (2005), 235-258.

[7] K. Dupoiron, P. Mathieu, and J. San Martin, Formule d'Itô pour des diffusions uniformément elliptiques, et processus de Dirichlet, Potential Anal. 21(1) (2004), 7-33.

[8] N. Eisenbaum, Integration with respect to local time, Potential Anal. 13(4) (2000), 303-328.

[9] N. Eisenbaum, On Itô's formula of Föllmer and Protter, in: "Séminaire de Probabilités, XXXV", Lecture Notes in Math. 1755, Springer, Berlin, 2001, pp. 390-395.

[10] F. Flandoli, F. Russo, and J. Wolf, Some SDEs with distributional drift. II. Lyons-Zheng structure, Itô's formula and semimartingale characterization, Random Oper. Stochastic Equations 12(2) (2004), 145-184.

[11] H. Föllmer, P. Protter, and A. N. Shiryayev, Quadratic covariation and an extension of Itô's formula, Bernoulli 1(1-2) (1995), 149-169. 
[12] R. Ghomrasni and G. Peskir, Local time-space calculus and extensions of Itô's formula, in: "High dimensional probability, III" (Sandjberg, 2002), Progr. Probab. 55, Birkhäuser, Basel, 2003, pp. $177-192$.

[13] S. Moret, Generalitzacions de la Formula d'Itô i estimacions per martingales, Ph.D. thesis, Universitat de Barcelona (1999).

[14] S. Moret And D. NuAlart, Quadratic covariation and Itô's formula for smooth nondegenerate martingales, J. Theoret. Probab. 13(1) (2000), 193-224.

[15] D. Nualart, "The Malliavin calculus and related topics", Second edition, Probability and its Applications (New York), SpringerVerlag, Berlin, 2006.

[16] D. NuAlart, Analysis on Wiener space and anticipating stochastic calculus, in: "Lectures on probability theory and statistics" (SaintFlour, 1995), Lecture Notes in Math. 1690, Springer, Berlin, 1998, pp. 123-227.

[17] L. C. G. Rogers And D. Williams, "Diffusions, Markov processes, and martingales", Vol. 2. Itô calculus, Reprint of the second (1994) edition, Cambridge Mathematical Library, Cambridge University Press, Cambridge, 2000.

Xavier Bardina:

Departament de Matemàtiques

Universitat Autònoma de Barcelona

08193-Bellaterra (Barcelona)

Spain

E-mail address: Xavier.Bardina@uab.cat

Carles Rovira:

Facultat de Matemàtiques

Universitat de Barcelona

Gran Via 585

08007-Barcelona

Spain

E-mail address: Carles.Rovira@ub.edu

Primera versió rebuda el 6 de novembre de 2008, darrera versió rebuda el 7 de maig de 2009. 